\title{
Superselective microcoil embolization \\ for endoscopically uncontrollable bleeding after endoscopic submucosal dissection
}

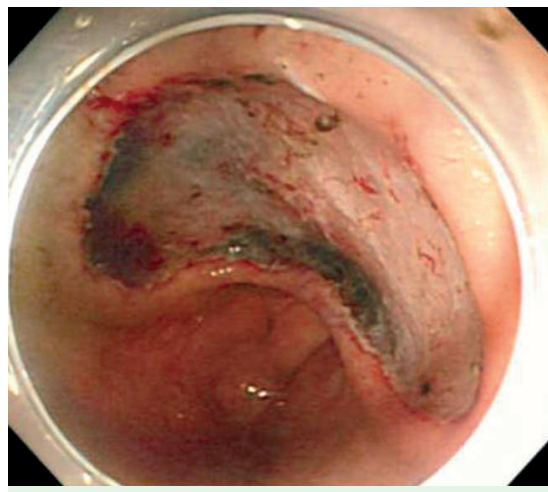

Fig. 1 Gastroscopic view showing a large artificial ulcer after endoscopic submucosal dissection.
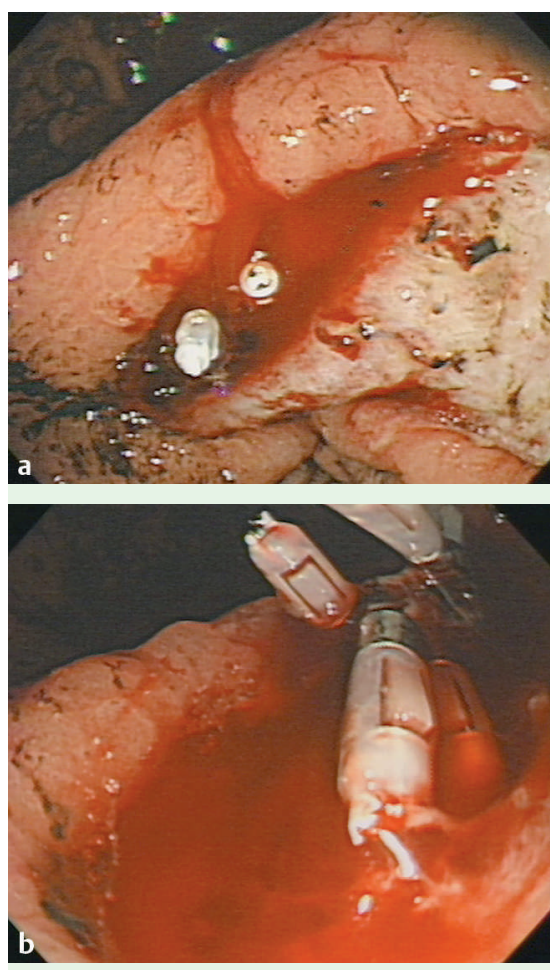

Fig. 2 Emergent gastroscopic views at 12 hours after endoscopic submucosal dissection: a ineffective hemostasis after two serial hemoclippings; and $\mathbf{b}$ close-up view of the bleeding focus - note the active, profuse oozing between the hemoclips.

A 57-year-old man underwent endoscopic submucosal dissection (ESD) for the treatment of early gastric cancer of the antrum ( $\bullet$ Fig. 1 ). There was no immediate bleeding after the procedure,

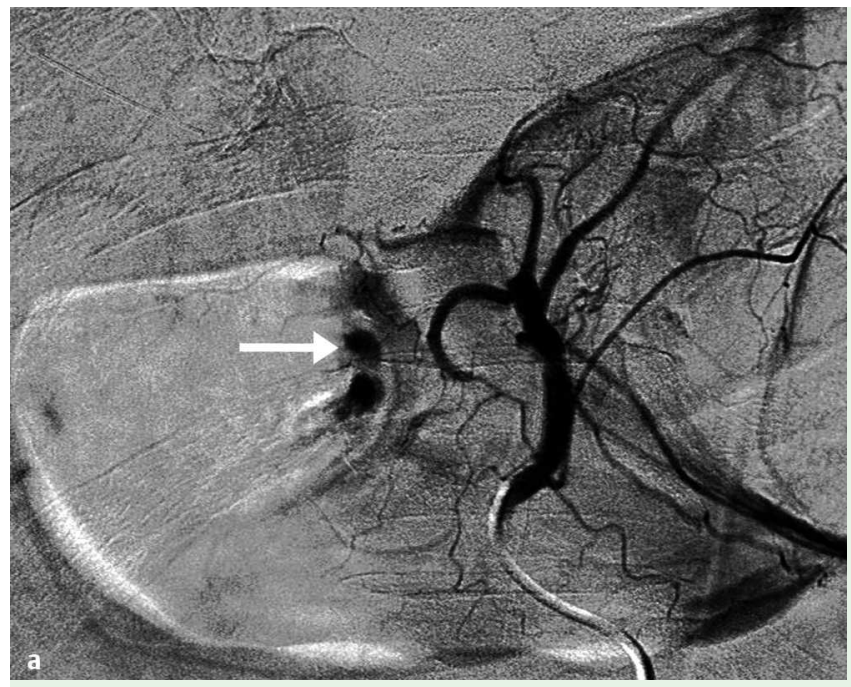

Fig. 3 a Superselective angiogram demonstrating extravasation, originating from a small side branch of the left gastric artery (arrow). b Final angiogram showing successful hemostasis after superselective placement of microcoils within the source artery (arrowheads).

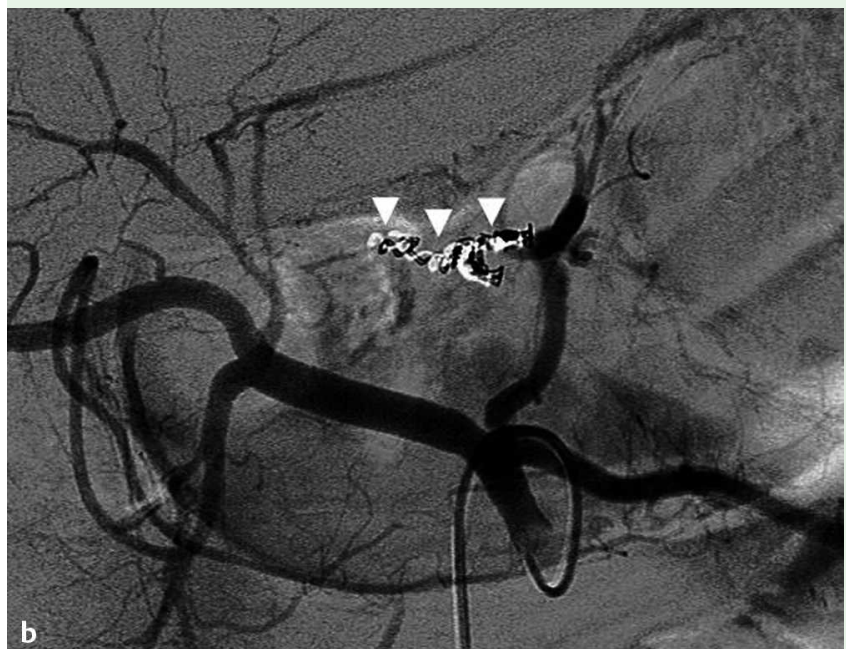

and a proton pump inhibitor was given intravenously for the prevention of delayed bleeding after ESD. Twelve hours after ESD, the patient suddenly developed hematemesis and melena. His systolic blood pressure was $80 \mathrm{~mm} \mathrm{Hg}$ and heart rate was 110 beats per minute. His hemoglobin level decreased from $14.5 \mathrm{~g} / \mathrm{dL}$ to $9.2 \mathrm{~g} / \mathrm{dL}$. Emergency gastroscopy revealed profuse oozing of blood from the artificial ulcer with an overlying clot. Endoscopic hemostasis using hemoclips was attempted, however, cessation of bleeding was not achieved as the active oozing masked its source ( Fig. 2 ).

Owing to hemodynamic instability, the patient underwent emergency angiography. Extravasation of contrast medium was seen from a small side branch of the left gastric artery in the antrum ( Fig. 3 a). The bleeding branch was successfully embolized by the placement of microcoils, both proximal and distal to the bleeding site, to prevent re-bleeding related to retrograde perfusion through the collateral vessels. No further extravasation of contrast medium was noted ( $\mathbf{F i g . 3 b}$ ). The patient was discharged after 5 days of close observation and conservative treatment. Endoscopy after 1 month revealed an ESD ulcer scar, without any evidence of ischemic complications ( Fig. 4).

Delayed bleeding, which is defined as hematemesis or melena at $0-30$ days after the procedure, may be the most se- 


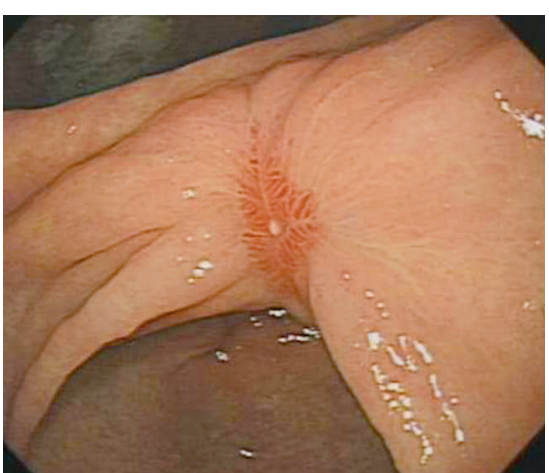

Fig. 4 Follow-up gastroscopic view at 30 days after endoscopic submucosal dissection (ESD) shows an ESD ulcer scar without evidence of ischemic complications.

rious complication of ESD [1]. Delayed recognition or failure to attempt endoscopic hemostasis for such an event may cause subsequent cardiovascular compromise. This report suggests that superselective microcoil embolization can be a lifesaving therapeutic option for endoscopically uncontrollable bleeding after ESD, and it can help avoiding emergency laparotomy [2].
Endoscopy_UCTN_Code_TTT_1AO_2AD

Endoscopy_UCTN_Code_TTT_1AO_2AG

C. K. Lee, J. Y. Park, T. H. Lee, S. H. Lee, I. K. Chung, S. H. Park, H. S. Kim, S. J. Kim Division of Gastroenterology, Department of Internal Medicine, Soonchunhyang University College of Medicine, Cheonan Hospital, Cheonan, Korea

\section{References}

1 Takizawa K, Oda I, Gotoda Tet al. Routine coagulation of visible vessels may prevent delayed bleeding after endoscopic submucosal dissection - an analysis of risk factors. Endoscopy 2008; 40: 179-183

2 Mezawa S, Homma H, Murase K et al. Superselective transcatheter embolization for acute lower gastrointestinal hemorrhage after endoscopic mucosal resection: a report of 3 cases. Hepatogastroenterology 2003; 50: $735-737$
Bibliography

DOI $10.1055 / \mathrm{s}-0029-1214627$

Endoscopy 2009; 41: E109-E110

(c) Georg Thieme Verlag KG Stuttgart · New York . ISSN 0013-726X

Corresponding author

\section{K. Lee, MD, PhD}

Division of Gastroenterology

Department of Internal Medicine

Soonchunhyang University College of Medicine

Cheonan Hospital

23-20 Bongmyung-dong

Cheonan

Choongnam 330-721

Korea

Fax: +82-41-5745762

cklee92@paran.com 